\title{
An effective coded excitation scheme based on a predistorted FM signal and an optimized digital filter
}

\author{
Misaridis, Thanasis; Jensen, Jørgen Arendt
}

Published in:

1999 IEEE Ultrasonics Symposium Proceedings

Link to article, DOI:

10.1109/ULTSYM.1999.849299

Publication date:

1999

Document Version

Publisher's PDF, also known as Version of record

Link back to DTU Orbit

Citation (APA):

Misaridis, T., \& Jensen, J. A. (1999). An effective coded excitation scheme based on a predistorted FM signal and an optimized digital filter. In 1999 IEEE Ultrasonics Symposium Proceedings (Vol. 1-2, pp. 1589-1593). IEEE. I E E E International Ultrasonics Symposium. Proceedings https://doi.org/10.1109/ULTSYM.1999.849299

\section{General rights}

Copyright and moral rights for the publications made accessible in the public portal are retained by the authors and/or other copyright owners and it is a condition of accessing publications that users recognise and abide by the legal requirements associated with these rights.

- Users may download and print one copy of any publication from the public portal for the purpose of private study or research.

- You may not further distribute the material or use it for any profit-making activity or commercial gain

- You may freely distribute the URL identifying the publication in the public portal 


\title{
An effective coded excitation scheme based on a predistorted FM signal and an optimized digital filter
}

\author{
Thanassis X. Misaridis and Jørgen A. Jensen \\ Center for Fast Ultrasound Imaging, Department of Information Technology, Build. 344, \\ Technical University of Denmark, DK-2800 Lyngby, Denmark
}

\begin{abstract}
This paper presents a coded excitation imaging system based on a predistorted FM excitation and a digital compression filter designed for medical ultrasonic applications, in order to preserve both axial resolution and contrast. In radars, optimal Chebyshev windows efficiently weight a nearly rectangular spectrum. For the small time-bandwidth (TB) products available in ultrasound, the rectangular spectrum approximation is not valid, which reduces the effectiveness of weighting. Additionally, the distant range sidelobes are associated with the ripples of the spectrum amplitude and, thus, cannot be removed by weighting. We show that by using a predistorted chirp with amplitude or phase shaping for amplitude ripple reduction and a correlation filter that accounts for the transducer's natural frequency weighting, output sidelobe levels of -35 to $-40 \mathrm{~dB}$ are directly obtained. When an optimized filter is applied on receive, contrast or resolution can be traded in for range sidelobe levels down to $-86 \mathrm{~dB}$. The digital filter is designed to efficiently use the available bandwidth and at the same time to be insensitive to the transducer's impulse response. For evaluation of the method, simulations were performed with the program Field II.
\end{abstract}

A commercial scanner (B-K Medical 3535) was modified and interfaced to an arbitrary function generator along with an RF power amplifier (Ritec). Hydrophone measurements in water were done to establish excitation voltage and corresponding intensity levels ( $\mathrm{I}_{s p t p}$ and $\mathrm{I}_{s p t a}$ ) well below the safety limits. In vivo images of the liver from two healthy volunteers show apparent increased depth of penetration of about $4 \mathrm{~cm}$ at $4 \mathrm{MHz}$, for codes of length $25 \mu \mathrm{s}$. Images taken from a non-attenuating wire phantom show that the $-20 \mathrm{~dB}$ axial resolution for the coded scheme is as good as with pulse excitation (about $1.5 \lambda$ ), depending on the filter design criteria. The axial sidelobes are below $-40 \mathrm{~dB}$, which is the noise level of the measuring imaging system. The proposed excitation/compression scheme shows good overall performance and stability to the frequency shift due to attenuation. It increases the penetration depth, and still yields a high resolution and low adjustable sidelobes.

\section{Introduction}

Coded excitation in medical ultrasound can be used for improving the signal-to-noise ratio (SNR) and/or the penetration depth, as long as both sidelobe level and energy are kept acceptably low for ultrasound imaging. An improvement in SNR of $10 \mathrm{~dB}$ or more is expected [1], [2], depending on the code parameters, and the available bandwidth. As we reported previously [2], clinical images of the liver from two healthy volunteers demonstrated an improvement in penetration depth of at least 4 to $5 \mathrm{~cm}$ at $4 \mathrm{MHz}$ with temporal sidelobe levels lower than $-35 \mathrm{~dB}$. In a high contrast imaging system this is not sufficient and this paper primarily focuses on an optimized design of pulse compression schemes applicable to ultrasound.

Linear FM waveforms have been used as coded excitation because of their important symmetry properties and their linear group delay. Non-linear chirps do not possess this linearity and therefore tend to be more sensitive to frequency shifts from the medium to be imaged. As it will be shown, linear chirps with amplitude tapering can give excellent performance, if they are designed properly. M-sequences and polyphase codes that have been used in radars are based on abrupt changes in phase that contain high harmonics, that the ultrasonic transducer smoothes out. Therefore, pulse compression with binary codes applied in ultrasound systems has worse performance than in radars due to the transducer, and have not been considered further.

\section{Mismatched filtering}

Range sidelobes represent an inherent part of the pulse compression mechanism, primarily due to the abrupt rise in the signal spectrum. In radar systems, the most used approach for sidelobe reduction is weighting either in the time or in the frequency domain. These approaches are not equivalent for low TB products. Time weighting is preferable and has been used exclusively in this paper, because it yields lower sidelobe compression outputs [3]. Mismatched filtering has been treated extensively in the radar literature [4], [5]. Briefly, the 
transfer function of time weighting-based mismatched filters is the complex conjugate of an amplitude-weighted version of the excitation signal. Among a plethora of window functions, the Dolph-Chebyshev window exhibits the minimum main-lobe width for a specified sidelobe level and has been used extensively in radars [5]. We have used other windows such as the Blackman-Harris [6] windows, which yield narrow mainlobe width with adequately low sidelobe levels. The drawbacks for the low sidelobe level are a) a broadening of the mainlobe and associated loss in axial resolution and b) a loss in SNR.

\section{The effect of the transducer}

In ultrasound, bandpass filtering of the excitation signal occurs inherently from the transducer. The transducer effect on pulse compression is illustrated in Fig. 1, based on measurements of the pulse-echo impulse response. The transducer used is a $4 \mathrm{MHz}$ single-element transducer (B-K Medical) and the calculated $-6 \mathrm{~dB}$ fractional bandwidth was $65 \%$. When the bandwidth of the chirp matches the transducer's bandwidth, the presence of the transducer reduces the near range sidelobes from -13.2 to $-32 \mathrm{~dB}$ below the mainlobe. The linear FM signal used for Fig. 1 had 1.2 higher bandwidth, and that reduces the sidelobes further down to $-42 \mathrm{~dB}$. When mismatched filtering is applied (bold lines of Fig. 1), near range sidelobes can be further reduced at the expense of an additional broadening in the main lobe. The effect of the transducer is equivalent to an additional weighting that, although suboptimal, is beneficial to sidelobe reduction. In radars, weighting of the transmitted pulse has been avoided in order to send out more energy and all processing is done on the receiver.

\section{Reduction of the Fresnel ripples}

As Fig. 1 shows, weighting severely fails to achieve the specified sidelobe levels in the $\pm T / 2$ region. This is an important consideration in the design of efficient coded excitation systems. The distant sidelobes increase the mainlobe-to-sidelobe energy ratio (MSR), an important image quality measure, which describes the leakage of energy from bright into dark areas of the image [7]. As it will be shown, the distant sidelobes are not related to the properties of the weighting function, but to the Fresnel ripples present in the spectrum of the FM signals. The efficiency of frequency windowing is based on the concept of shaping a rectangular spectrum to reduce the ripples of the sinc function in the time domain. For TB products larger than 50, the linear FM signal has approximately a rectangular envelope and a quadratic phase in both time and frequency domain, since the instantaneous frequency and group delay are linear functions of time and frequency respectively. This approximation is not valid for the low TB chirps that can be used in medical ultrasound. The
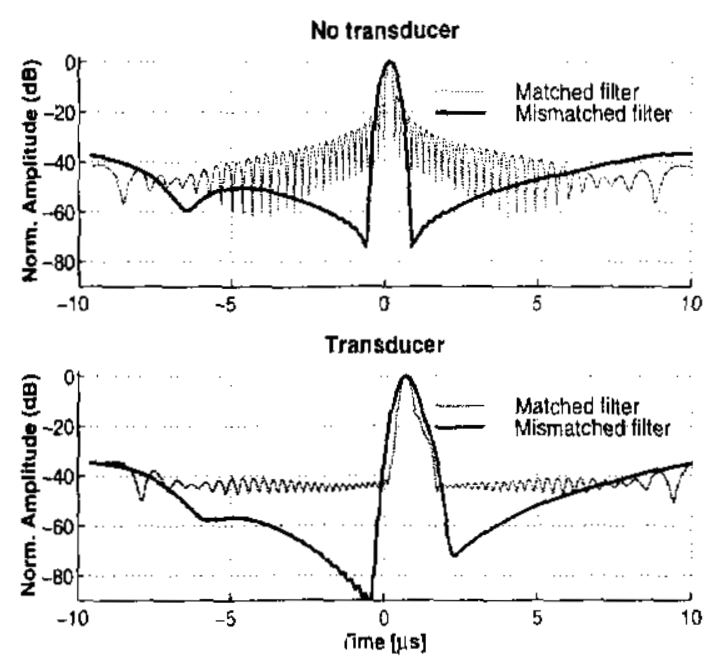

Figure 1: The effect of the transducer on pulse compression. The faint lines are the matched-filter outputs and the bold lines are the outputs when a Dolph-Chebyshev window has been applied to the compression filter. The specified sidelobe level for the window was $-90 \mathrm{~dB}$.

exact solution for the spectrum is given by [8]:

$$
S(f)=\frac{1}{\sqrt{2 B}} e^{-j \pi f^{2} T / B}\left\{C\left(x_{1}\right)+C\left(x_{2}\right) \pm j\left[S\left(x_{1}\right)+S\left(x_{2}\right)\right]\right\}
$$

where $T$ is the duration of the FM signal, $B$ its bandwidth, $D=T B, x_{1}=\sqrt{2 D}\left(\frac{1}{2}-\frac{f}{B}\right)$ and $x_{2}=\sqrt{2} \bar{D}\left(\frac{1}{2}+\frac{f}{B}\right) . C$ and $S$ are the quasi-oscillating Fresnel integrals that are responsible for the ripples in both spectrum amplitude and phase. The distant range sidelobes are paired-echo distortions due to deviation from the ideal rectangular spectrum amplitude and, thus, are not affected by any frequency weighting. This can be understood if the following Fourier pair is considered [5]:

$\frac{a_{n}}{2} g\left(t+\frac{n}{B}\right)+g(t)+\frac{a_{n}}{2} g\left(t-\frac{n}{B}\right) \stackrel{I}{\longrightarrow} G(f)\left[1+a_{n} \cos \left(2 \pi n \frac{f}{B}\right)\right]$

The presence of $n$ spectrum amplitude ripples of amplitude $a_{n}$ over the passband $B$ of a signal spectrum $G(f)$ create symmetrical paired echoes in the time domain delayed and advanced from the main signal by $n / B$ and scaled in amplitude by $a_{n} / 2$. In a linear $F M$ signal, the number of ripples and their amplitude depend on the TB product. In our case, the approximate paired-echo sidelobes are at about $\pm T / 2$ relative to the center of the compressed pulse with an amplitude of about -30 to $-40 \mathrm{~dB}$ [9]. The displayed dynamic range of a typical ultrasound image is $50 \mathrm{~dB}$ or greater, and therefore methods that compensate for the distant axial sidelobes have to be employed.

We have tested three different approaches for ripple reduction: envelope \& phase modulation, phase predistortion, and time weighting of the edges. 

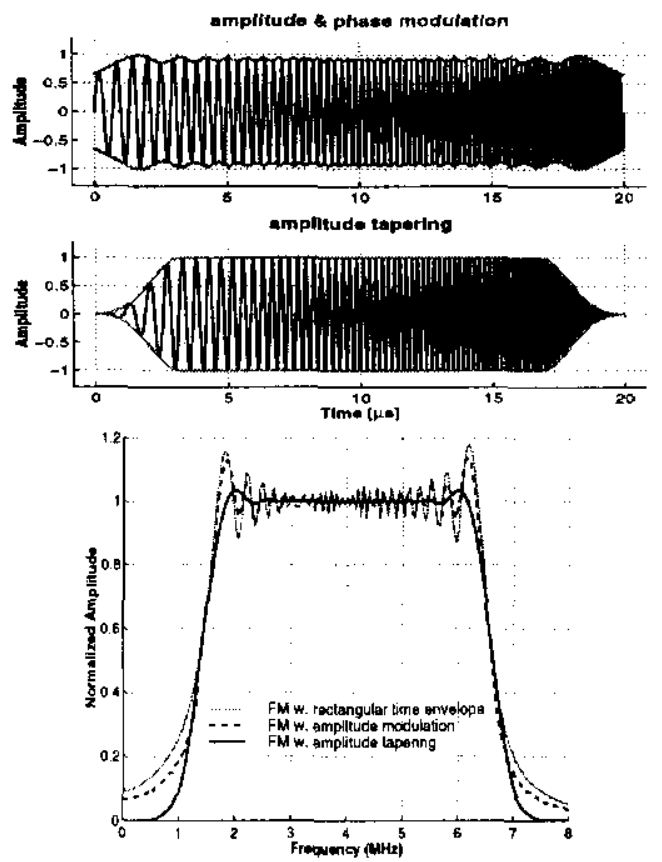

Figure 2: Reduction of spectrum ripples using envelope \& phase modulation and amplitude tapering.

Envelope modulation. This approach is based on the symmetry property of the linear FM signal in time and frequency. A linear FM signal with a constant amplitude envelope in the time domain yields a rectangular amplitude spectrum with ripple distortion. The reciprocal signal with a constant amplitude spectrum and strictly quadratic phase will have an amplitude and phase in the time domain given by Eq.(1), if $T$ is replaced with $B$ and $t$ with $f[8]$.

Phase predistortion. An alternative approach with similar performance is to use a phase predistortion of the transmitted signal, since amplitude and phase distortions have functional similarity and produce similar effects [9]. Both quadratic and cubic predistortion functions have been previously reported [9],[3]. Phase predistorted chirps have the advantage of sending out more energy, but are more vulnerable to phase distortions added from acoustic propagation.

Amplitude tapering. The amplitude ripples of the spectrum can be attributed to the sharp rise-time of the time envelope, since a linear FM signal with infinite duration has no ripples. Data correlating pulse rise-time and spectrum ripple have been previously reported [9]. Based on this analysis, a modified chirp with amplitude tapering of the transmitted signal has been generated. The attainable ripple reduction is a function of the signal's bandwidth, the choice of the tapering function and its duration. Fig. 2 shows the results of the different approaches on spectrum ripple reduction. The tapered function used in Fig. 2 is a Tukey window with a duration of
$0.15 \mathrm{~T}$.

\section{Waveform and filter design}

As it was shown in the previous section, amplitude tapering is the most efficient way to reduce the Fresnel ripples of the spectrum, if the power amplifier allows control of the transmitted pulse rise time. For a given duration $T$, the design parameters are

i) the frequency band that is swept relative to the transducer's bandwidth

ii) the choice of the tapering function and its duration

iii) the choice of the weighting function.
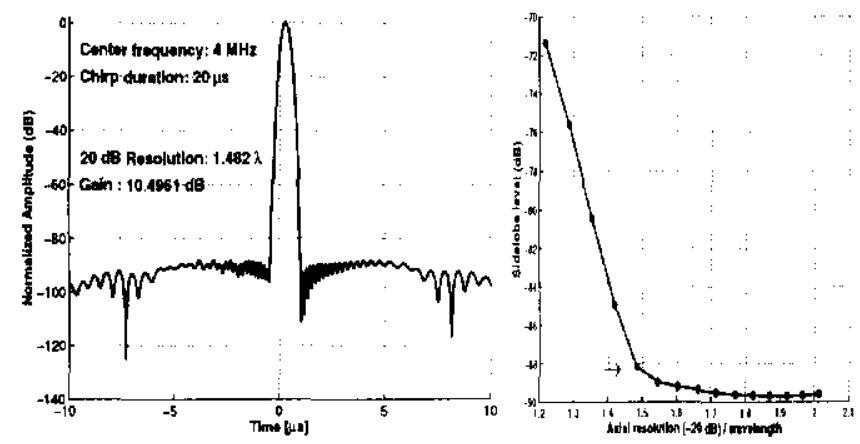

Figure 3: Optimized compression output. The right graph shows the tradeoffs between sidelobe level and axial resolution for a Dolph-Chebyshev mismatched compression filter. For most applications, the appropriate choice is at points in the knees of the curve as the one indicated by the arrow.

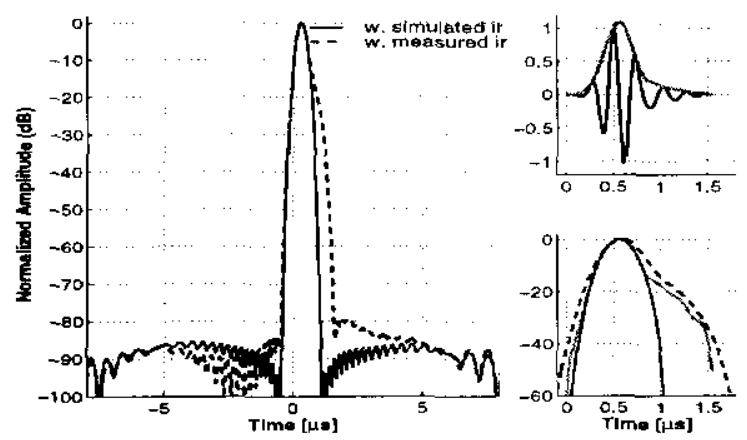

Figure 4: The effect of the transducer on the new scheme. The gray lines are the envelope of the measured impulse response and the dotted lines is the compressed output when the actual transducer is used. The black solid line in the bottom right figure is the logarithmic envelope of the simulated transducer.

The transducer's bandwidth can be modeled, but the phase 
of jts transfer function is unknown and, thus, can not be compensated for. The effort here is to minimize the effect of the convolution between the transducer impulse response and the excitation signal on the design of the waveforms and the compression filter. This is done in the excitation signal by sweeping a bandwidth slightly larger than the transducer's frequency passband. This has an additional advantage: the effect of the two main rise and fall overshoots of the spectrum (see Fig. 2) is minimized. With this design, the range sidelobes can be as low as -88.2 dB. Fig. 3 and Fig. 5 show optimized compression outputs for different frequencies and signal durations. The compression scheme is rather insensitive to the actual impulse response of the transducer (Fig. 4). The increased bandwidth of the excitation waveform also yields a better axial resolution and higher gain. The appropriate choice of the weighting function is a tradeoff between axial resolution and sidelobe levels (Fig. 3). If the length of the excitation signal is doubled, the sidelobes drop down to $-105.5 \mathrm{~dB}$ with a superb resolution of $1.45 \lambda$ (Fig. 5). For comparisons, the $-20 \mathrm{~dB}$ axial resolution with a typical pulse excitation is $1.46 \lambda$. The design gives even better results for higher frequencies.
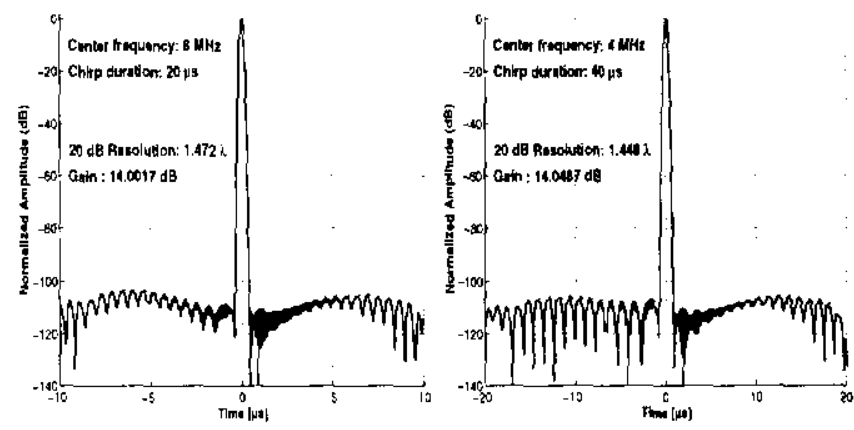

Figure 5: Optimized compression outputs for FM signals with amplitude tapering.

\section{Simulated and experimental results}

The new excitation/compression scheme has been tested with the simulation program Field II [10]. The simulated image of Fig. 6 shows good performance when attenuation is present.

An experimental coded excitation system has been used for the evaluation of the new method. A commercial scanner (B$K$ Medical 3535) was used along with a single-element mechanically rotating $4 \mathrm{MHz}$ transducer. A detailed description and a block diagram of the system are given in [2]. The transmitter of the scanner was inactivated and the machine was modified to operate with external excitation. A LeCroy 9112 arbitrary function generator (AFG) was used for the generation of the coded waveforms on the computer. A power RF
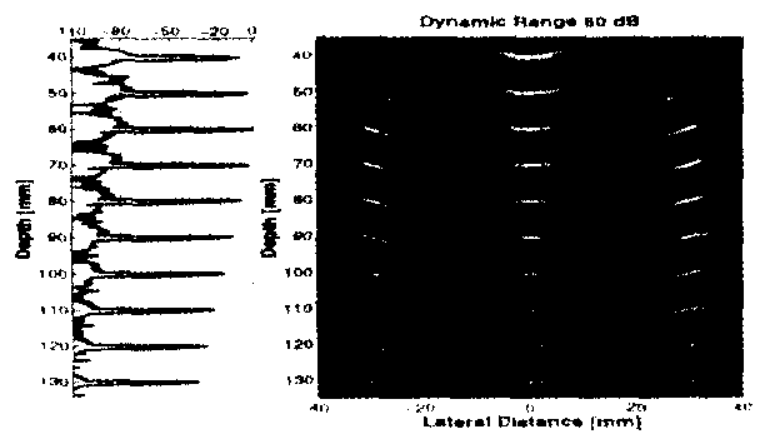

Figure 6: Simulated image of a phantom consisting of point scatterers in an attenuating medium with attenuation of 0.7 $\mathrm{dB} /[\mathrm{MHz} \times \mathrm{cm}]$. On the left of the graph, the central rf-line is plotted.

amplifier (Ritec 5000), specifically designed to drive ultrasound transducers, was used subsequently for amplification. The acquisition of the RF data was done using the sequential acquisition capabilities of an 8-bit digital oscilloscope (LeCroy LC334AM) at a minimum sampling sampling of 20 $\mathrm{MHz}$ depending on the requested depth of penetration and number of scan lines.

A linear FM signal $20 \mu$ s long was used, with a theoretical compression output as the one shown in Fig. 3. The power amplifier distorted the phase of the excitation signal, introducing spectral ripples. In order to evaluate the effect of this distortion, additional simulations were performed, in which the actual output of the power amplifier has been used. To simulate the imaging system further, the measured impulse response of the transducer has been used. A phantom was scanned using the experimental coded system. The phantom consisted of wires placed every $1 \mathrm{~cm}$ in water. Additional wires were placed off-axis for evaluation of the system's resolution. The resulting image is shown in Fig. 7. For quantitative comparisons, the rf-lines are also shown. There is an apparent agreement between simulated and experimental results. Fig. 7 also shows how the SNR improves from pulse to coded excitation (top graphs).

Acquired clinical images are shown in Fig. 8.

\section{Conclusions}

We have presented a new coded excitation scheme with distinct features, that make it attractive to the implementation of high-performance coded ultrasound systems. The range resolution that can be achieved is comparable to that of a conventional system, depending on the available bandwidth. The range sidelobes, at the same time, are well-below the limits of the typical dynamic range of an ultrasound image. The energy of the sidelobe region is also reduced by lowering the 

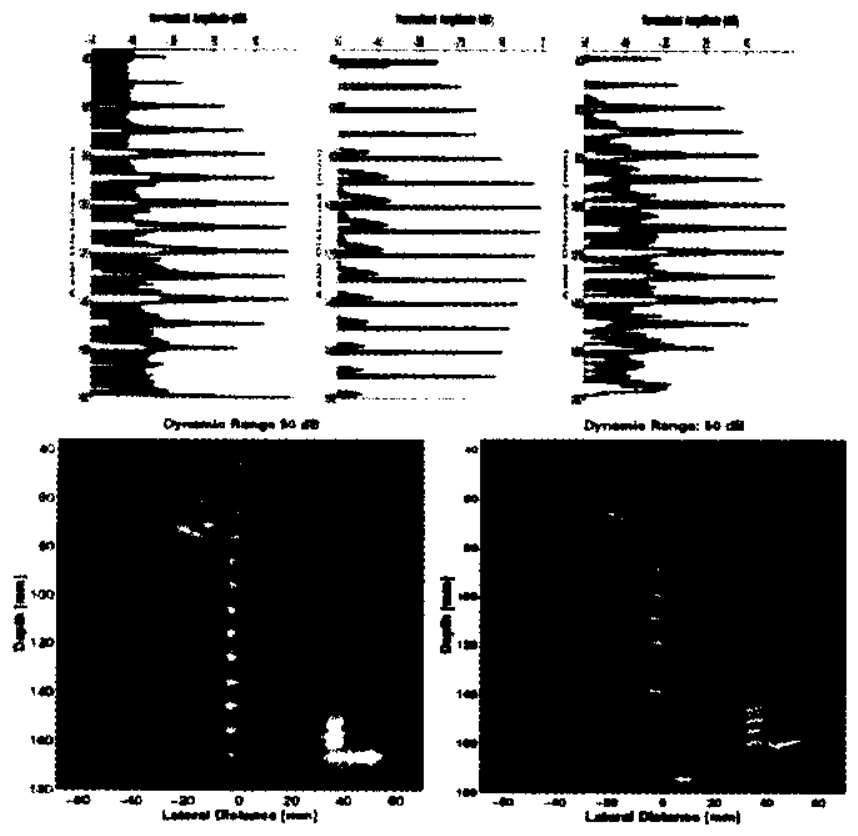

Figure 7: Acquired image of a wire phantom before and after compression. On the top of the graph, the central rf-lines are plotted from a) acquired image of the phantom with pulse excitation (not shown here), b) simulated image with coded excitation when the signal from the output of the power amplifier and the measured impulse response of the transducer have been used, and c) acquired image with coded excitation shown here.

distant sidelobes caused by the ripples of the spectrum's amplitude. Testing of the new coding excitation/compression scheme with Field II has shown good stability to the effect of frequency shift due to attenuation. Phantom images verified the good performance of the system. Compared to previously reported excitation systems, the proposed approach exhibits lower sidelobe energy (and therefore higher image contrast capabilities) and high compression gain, while maintaining axial resolution.

\section{Acknowledgments}

The authors would like to thank Kim Gammelmark, Christian H. Jørgensen, Niklas Lindberg, and Anders H. Thomsen for their work on the experimental setup, Dr. Morten H. Pedersen for some clinical scans and Ph.D. student Peter Munk for his valuable advices.

This work was supported by grant 9700883 and 9700563 from the Danish Science Foundation and by B-K Medical $\mathrm{A} / \mathrm{S}$.
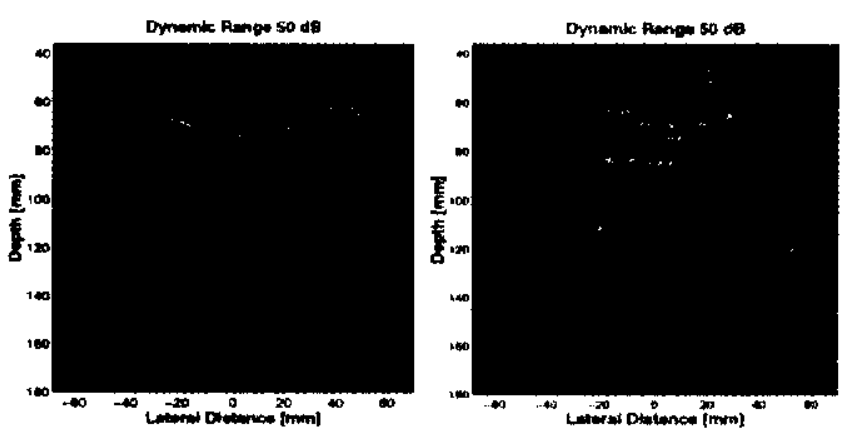

Figure 8: Acquired clinical images with pulse and coded excitation.

\section{References}

[1] M. O'Donnell. Coded excitation system for improving the penetration of real-time phased-array imaging systems. IEEE Trans. Ultrason., Ferroelec., Freq. Contr., 39:341-351, 1992.

[2] T. Misaridis, K. Gammelmark, C. H. Jørgensen, N. Lindberg, A. H. Thomsen, M. H. Pedersen, and J. A. Jensen. Potential of coded excitation in medical ultrasound imaging. Presented at the Ultrasonics International 1999. To be published in Ultrasonics.

[3] M. Kowatsch and H. R. Stocker. Effect of Fresnel ripples on sidelobe suppression in low time-bandwidth product linear FM pulse compression. IEE Proc., 129:41-44, 1982.

[4] C. E. Cook and M. Bernfeld. Radar signals. Artech House, Inc., 1993.

[5] M. I. Skolnik. Radar Handbook. McGraw-Hill, New York, second edition, 1990.

[6] F. J. Harris. On the use of windows for harmonic analysis with the discrete Fourier transform. Proc. IEEE, $66: 51-83,1978$.

[7] J. W. Adams. A new optimal window. IEEE Trans. Sig. Pro., 39:1753-1769, 1991.

[8] A. W. Rihaczek. Principles of high-resolution radar. McGraw-Hill, New York, 1969.

[9] C. E. Cook and J. Paolillo. A pulse compression predistortion function for efficient sidelobe reduction in a high-power radar. Proc. IEEE, 52:377-389, 1964.

[10] J. A. Jensen. Field: A program for simulating ultrasound systems. Med. Biol. Eng. Comp., 10th NordicBaltic Conference on Biomedical Imaging, Vol. 4, Supplement 1, Part 1:351-353, 1996b. 